\title{
Enhanced dispersion interaction in confined geometry
}

\author{
Michal Marcovitch ${ }^{1}$ and Haim Diamant ${ }^{2}$, * \\ ${ }^{1}$ School of Physics \& Astronomy \\ ${ }^{2}$ School of Chemistry \\ Raymond and Beverly Sackler Faculty of Exact Sciences, Tel Aviv University, Tel Aviv 69978, Israel
}

(Dated: October 2, 2005)

\begin{abstract}
The dispersion interaction between two point-like particles confined in a dielectric slab between two plates of another dielectric medium is studied within a continuum (Lifshitz) theory. The retarded (Casimir-Polder) interaction at large interparticle distances is found to be strongly enhanced as the mismatch between the dielectric permittivities of the two media is increased. The large-distance interaction is multiplied due to confinement by a factor of $\left(33 \gamma^{5 / 2}+13 \gamma^{-3 / 2}\right) / 46$ at zero temperature, and by $\left(5 \gamma^{2}+\gamma^{-2}\right) / 6$ at finite temperature, $\gamma=\epsilon_{\text {in }}(0) / \epsilon_{\text {out }}(0)$ being the ratio between the static dielectric permittivities of the inner and outer media. This confinement-induced amplification of the dispersion interaction can reach several orders of magnitude.
\end{abstract}

PACS numbers: 34.20.-b, 03.65.Sq, 82.70.-y

The dispersion interaction acts between any two polarizable objects, thus being one of the most ubiquitous interactions in nature 1, 2]. It plays a central role in numerous phenomena in chemical physics and materials science, including gas-liquid condensation, capillarity []. inter-surface interactions [4], and colloid stability [5].

The dispersion interaction is a quantum fluctuationinduced coupling between two polarizable particles mediated by the electromagnetic field. In an unconfined system at zero temperature there is a single length scale with which the interparticle distance $R$ is to be compared, i.e., the characteristic wavelength $\lambda_{0}$ of photon absorption by the particles, typically in the ultraviolet to visible range. London's calculation [6], valid in the nonretarded limit $R \ll \lambda_{0}$, yields the potential $U(R)=-\left[(3 \hbar / \pi) \int_{0}^{\infty} d \xi \alpha^{2}(i \xi)\right] R^{-6}$, where $\alpha(\omega)$ is the frequency-dependent polarizability of the particles. Casimir and Polder 7] recast the problem in quantumelectrodynamic terms, whereby the interaction arises from the effect of the particles on the zero-point modes of the electromagnetic field. For $R \ll \lambda_{0}$ the CasimirPolder result coincides with London's, yet in the retarded limit, $R \gg \lambda_{0}$, the interaction decays as $R^{-7}$, $U(R)=-\left[(23 /(4 \pi)) \hbar c \alpha^{2}(0)\right] R^{-7}[$ 8]. As a result, the dispersion interaction between two particles in the retarded regime (typically $R>0.1 \mu \mathrm{m}$ ) is extremely weak and has not been directly observed. (Particle-surface and surface-surface interactions across such micron-scale distances are much stronger and were successfully measured in the 1990s [9, 10].) In the current Letter we demonstrate that this weak particle-particle interaction can be dramatically amplified in confined geometries.

At a finite temperature $T$ another length scale appears, i.e., the thermal wavelength $\lambda_{T}=\hbar c / T \simeq 7.6 \mu \mathrm{m}$ at room temperature. (The Boltzmann constant is set hereafter to unity.) As was shown in Ref. [11], retardation and finite-temperature effects are intertwined. For $R \gg \lambda_{T}$, the Helmholtz free energy of interaction returns to a $R^{-6}$ dependence, $F(R)=-3 T \alpha^{2}(0) R^{-6}$.

The theory of dispersion interactions was extended by Lifshitz et al. to the case where the interacting objects as well as the intervening space are continuous media 12, 13. In this continuum theory the material response to electromagnetic fields is assumed to be fully captured by the complex, frequency-dependent dielectric permittivity $\epsilon(\omega)$ 14]. The current work is based on such a continuum approach. Despite the strong underlying assumption (after all, the media themselves consist of discrete polarizable particles), the Lifshitz theory has been widely used and experimentally corroborated [4]. It is expected to yield valid results so long as the distance between the two particles is much larger than the intermolecular distances in the materials.

In various circumstances particles are spatially confined, e.g., in porous media, micro-cavities, biological constrictions or nanofluidic devices. Such confinement introduces a new length scale, the separation $h$ between the bounding surfaces. The dispersion interaction between a single particle and confining surfaces has been extensively studied in the context of cavity QED [9, 15]. The effect of confinement on the interaction between two particles, however, has been only partially addressed. Confinement by two metallic plates (i.e., the limit where the permittivity of the outer medium $\epsilon_{\text {out }} \rightarrow \infty$ ) was found to drastically affect the interaction between two point-like particles in vacuum at zero [16] and nonzero [17] temperatures. The nonretarded interaction between particles confined by two dielectric plates at $T=0$ was addressed within a single-image approximation in Ref. 18]. We follow the lines of Mahanty, Ninham, Boström and Longdell [16, 17] and extend their theory to the general and more practical case of arbitrary permittivities of both inner and outer media, discovering a dramatic enhancement for experimentally relevant values of $\epsilon_{\text {in }}$ and $\epsilon_{\text {out }}$.

The system under consideration is schematically shown in Fig. 1 Two point-like, isotropic particles of polariz- 
ability $\alpha(\omega)$ are embedded in a slab of thickness $h$ and dielectric permittivity $\epsilon_{\text {in }}(\omega)$. The slab is bounded by two semi-infinite media of dielectric permittivity $\epsilon_{\text {out }}(\omega)$. We use cylindrical coordinates, $\mathbf{r}=(\rho, \varphi, z)$, the $\hat{\mathbf{z}}$ axis taken perpendicular to the bounding surfaces. For simplicity we specialize to the symmetric case where the particles lie on the slab midplane, $z=h / 2$, connected by the vector $\mathbf{R}=(R, 0,0)$. All materials are assumed nonmagnetic.

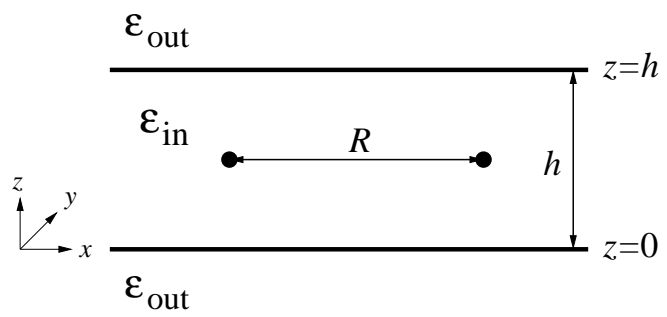

FIG. 1: Schematic view of the system and its parameters.

We employ the semiclassical scheme introduced by Mahanty and Ninham, which accurately reproduces the London and Casimir-Polder results [19]. In this theory the energy of interaction at $T=0$ is given by 1$]$

$$
\begin{aligned}
& T=0: \quad U(\mathbf{R})=-8 \pi \hbar \int_{0}^{\infty} d \xi \frac{\xi^{4}}{c^{4}} \alpha^{2}(i \xi) \\
& \times \operatorname{Tr}[\mathbf{G}(\mathbf{R}, i \xi) \mathbf{G}(-\mathbf{R}, i \xi)],
\end{aligned}
$$

and the Helmholtz free energy at finite $T$ by

$$
\begin{aligned}
& T>0: \quad F(\mathbf{R})=-(4 \pi)^{2} T \sum_{n=0}^{\infty} \alpha^{2}\left(i \xi_{n}\right)\left(\xi_{n}^{4} / c^{4}\right) \\
& \times \operatorname{Tr}\left[\mathbf{G}\left(\mathbf{R}, i \xi_{n}\right) \mathbf{G}\left(-\mathbf{R}, i \xi_{n}\right)\right], \quad \xi_{n}=\left(2 \pi c / \lambda_{T}\right) n,
\end{aligned}
$$

the prime indicating that the $n=0$ term is multiplied by 1/2. In Eqs. (1) and (2) $\mathbf{R}=\mathbf{r}-\mathbf{r}^{\prime}$, and $\mathbf{G}\left(\mathbf{r}, \mathbf{r}^{\prime}, \omega\right)$ is the dyadic Green tensor of the electric-field wave equation,

$$
\nabla \times \nabla \times \mathbf{G}-\epsilon_{m}(\omega)\left(\omega^{2} / c^{2}\right) \mathbf{G}=\mathbf{I} \delta\left(\mathbf{r}-\mathbf{r}^{\prime}\right),
$$

where $\mathbf{I}$ is the identity tensor and $\epsilon_{m}=\epsilon_{\text {in }}$ or $\epsilon_{\text {out }}$ depending on whether $\mathbf{r}$ lies in the inner or outer medium. (The position $\mathbf{r}^{\prime}$ is taken inside the slab.) The boundary conditions in the current case are continuity across the bounding surfaces of the tangential components of both the electric and magnetic fields. This imposes continuity on $\hat{\mathbf{z}} \times \mathbf{G}$ and $\hat{\mathbf{z}} \times \nabla \times \mathbf{G}$ across $z=0$ and $z=h$.

Thus, this scheme reduces the problem to finding the Green tensor $\mathbf{G}\left(\mathbf{r}, \mathbf{r}^{\prime}, \omega\right)$ of Eq. (3) with the aforementioned boundary conditions. The derivation is technically complicated and can be found in Refs. 20, 21]. In the symmetric case of interest, $\mathbf{r}^{\prime}=(0,0, h / 2)$ and
$\mathbf{r}=(R, 0, h / 2)$, the tensor becomes diagonal,

$$
\begin{gathered}
\frac{\xi^{2}}{c^{2}} G_{i j}(R, i \xi)=\frac{1}{4 \pi \epsilon_{\mathrm{in}}(i \xi) R^{3}} \int_{0}^{\infty} d x g_{i}(R, i \xi, x) \delta_{i j} \\
g_{\rho}=\left[q^{2} x\left(e^{v}-t\right)\left(e^{v}-u\right) J_{0}(x)-\left(x^{2}\left(e^{2 v}+t u\right)\right.\right. \\
\left.\left.\quad-e^{v}\left(p^{2}+q^{2}\right)(t+u)\right) J_{1}(x)\right] /\left[q\left(e^{v}-t\right)\left(e^{v}+u\right)\right] \\
g_{\varphi}=\left[p^{2} x\left(e^{v}+t\right)\left(e^{v}+u\right) J_{0}(x)+\left(x^{2}\left(e^{2 v}+t u\right)\right.\right. \\
\left.\left.-e^{v}\left(p^{2}+q^{2}\right)(t+u)\right) J_{1}(x)\right] /\left[q\left(e^{v}-t\right)\left(e^{v}+u\right)\right] \\
g_{z}=-x^{3}\left(e^{v}+u\right) J_{0}(x) /\left[q\left(e^{v}-u\right)\right],
\end{gathered}
$$

where $J_{k}(x)$ are Bessel functions, and the following abbreviations have been used: $\gamma=\epsilon_{\text {in }} / \epsilon_{\text {out }}, p=R \xi \epsilon_{\text {in }}^{1 / 2} / c$, $q=\left(x^{2}+p^{2}\right)^{1 / 2}, s=\left(x^{2}+p^{2} / \gamma\right)^{1 / 2}, t=(q-s) /(q+s)$, $u=(q-\gamma s) /(q+\gamma s)$, and $v=h q / R$. Given $\epsilon_{\text {in,out }}(i \xi)$ and $\alpha(i \xi)$, one can substitute Eq. (4) in Eq. (1) or (2) and calculate numerically the interaction potential.

It is instructive, however, to first analyze the interaction in several asymptotic limits. We begin with the small-distance limit, recovering the known results for unconfined particles. For $R \ll h$ the expressions for $g_{i}$ are expanded to leading order in large $v$, whereupon the integration in Eq. (4) can be carried out analytically. Substituting the result in Eq. (11), we get $U(R)=-(\hbar / \pi) R^{-6} \int_{0}^{\infty} d \xi e^{-2 p}\left(3+6 p+5 p^{2}+2 p^{3}+\right.$ $\left.p^{4}\right) \alpha^{2}(i \xi) / \epsilon_{\text {in }}^{2}(i \xi)$. In the nonretarded limit, $R \ll \lambda_{0}$, we take the leading order in small $p$, for which the London result is recovered,

$$
T=0, R \ll h, \lambda_{0}: U(R)=-\frac{3 \hbar}{\pi R^{6}} \int_{0}^{\infty} d \xi \frac{\alpha^{2}(i \xi)}{\epsilon_{\mathrm{in}}^{2}(i \xi)},
$$

with the appropriate correction due to the fact that the particles are not in vacuum $\left(\epsilon_{\text {in }} \neq 1\right)$. In the retarded limit, $R \gg \lambda_{0}$, the integral over $\xi$ is dominated by small (static) $\xi$. An asymptotic analysis then recovers the Casimir-Polder result with the correction for $\epsilon_{\text {in }} \neq 1$,

$$
T=0, \lambda_{0} \ll R \ll h: U(R)=-\frac{23 \hbar c \alpha^{2}(0)}{4 \pi \epsilon_{\mathrm{in}}^{5 / 2}(0) R^{7}} .
$$

Similarly, substitution of the asymptotic Green tensor for $R \ll h$ in Eq. (2) yields $F(R)=-2 T R^{-6} \sum_{n=0}^{\prime \infty} e^{-2 p_{n}}\left(3+6 p_{n}+5 p_{n}^{2}+2 p_{n}^{3}+\right.$ $\left.p_{n}^{4}\right) \alpha^{2}\left(i \xi_{n}\right) / \epsilon_{\text {in }}^{2}\left(i \xi_{n}\right)$, where $p_{n}=R \xi_{n} \epsilon_{\text {in }}^{1 / 2} / c$. Subsequently taking the limit $R \ll \lambda_{T}$, we expand to leading order in small $p_{n}$ [22] and recover the known result for the nonretarded interaction at finite temperature [23],

$$
T>0, R \ll h, \lambda_{T}: F(R)=-\frac{6 T}{R^{6}} \sum_{n=0}^{\infty} \frac{\alpha^{2}\left(i \xi_{n}\right)}{\epsilon_{\mathrm{in}}^{2}\left(i \xi_{n}\right)} .
$$

In the other limit, $R \gg \lambda_{T}$, the sum is dominated by the $n=0$ term, leading to the known retarded interaction at finite temperature 11],

$$
T>0, \lambda_{T} \ll R \ll h: F(R)=-\frac{3 T \alpha^{2}(0)}{\epsilon_{\mathrm{in}}^{2}(0) R^{6}} .
$$


We now turn to the more interesting asymptotic limit of large $R$ where confinement sets in. In the limit $R \gg h$ the expressions for $g_{i}$ in Eq. (4) are expanded to leading order in small $v$, and the integration in Eq. (4) is performed analytically. The result is substituted in Eq. (11) to yield $U(R)=-(\hbar /(2 \pi)) R^{-6} \int_{0}^{\infty} d \xi e^{-2 \bar{p}}[(5+$ $\left.\gamma^{-4}\right)+\left(10+2 \gamma^{-4}\right) \bar{p}+\left(7+3 \gamma^{-4}\right) \bar{p}^{2}+\left(2+2 \gamma^{-4}\right) \bar{p}^{3}+(1+$ $\left.\left.\gamma^{-4}\right) \bar{p}^{4}\right] \alpha^{2}(i \xi) / \epsilon_{\text {out }}^{2}(i \xi)$, where $\bar{p}=\gamma^{-1 / 2} p$. In the nonretarded regime, this expression is expanded to leading order in small $\bar{p}$, leading to

$$
\begin{aligned}
& T=0, h \ll R \ll \lambda_{0}: \\
& U(R)=-\frac{3 \hbar}{\pi R^{6}} \int_{0}^{\infty} d \xi \frac{\alpha^{2}(i \xi)}{\epsilon_{\mathrm{in}}^{2}(i \xi)} \frac{5 \gamma^{2}(i \xi)+\gamma^{-2}(i \xi)}{6} .
\end{aligned}
$$

Equation (9) gives the nonretarded interaction under confinement. It reduces to the London result, Eq. ([5), for $\gamma=\epsilon_{\text {in }} / \epsilon_{\text {out }}=1$. This result has a rather restricted validity as it requires that $h$ be much smaller than $\lambda_{0}$. (A detailed discussion of the confined nonretarded interaction will be given elsewhere.) In the retarded regime the integral is dominated by small $\xi$, leading to

$$
\begin{aligned}
& T=0, R \gg h, \lambda_{0}: \\
& U(R)=-\frac{23 \hbar c \alpha^{2}(0)}{4 \pi \epsilon_{\mathrm{in}}^{5 / 2}(0) R^{7}} \frac{33 \gamma^{5 / 2}+13 \gamma^{-3 / 2}}{46},
\end{aligned}
$$

where we have written $\gamma(0)=\gamma$ for brevity. This retarded interaction under confinement converges to the Casimir-Polder expression, Eq. (6), for $\gamma=1$.

At finite temperature we substitute the asymptotic Green tensor for $R \gg h$ in Eq. (2), resulting in $F(R)=$ $-T R^{-6} \sum_{n=0}^{\prime \infty} e^{-2 \bar{p}_{n}}\left[\left(5+\gamma^{-4}\right)+\left(10+2 \gamma^{-4}\right) \bar{p}_{n}+(7+\right.$ $\left.\left.3 \gamma^{-4}\right) \bar{p}_{n}^{2}+\left(2+2 \gamma^{-4}\right) \bar{p}_{n}^{3}+\left(1+\gamma^{-4}\right) \bar{p}_{n}^{4}\right] \alpha^{2}\left(i \xi_{n}\right) / \epsilon_{\text {out }}^{2}\left(i \xi_{n}\right)$, where $\bar{p}_{n}=R \xi_{n} \epsilon_{\text {out }}^{1 / 2} / c$. For $R \ll \lambda_{T}$ we take the leading order in small $\bar{p}_{n}[22]$ and get

$$
\begin{aligned}
& T>0, h \ll R \ll \lambda_{T}: \\
& F(R)=-\frac{6 T}{R^{6}} \sum_{n=0}^{\prime} \frac{\alpha^{2}\left(i \xi_{n}\right)}{\epsilon_{\mathrm{in}}^{2}\left(i \xi_{n}\right)} \frac{5 \gamma^{2}\left(i \xi_{n}\right)+\gamma^{-2}\left(i \xi_{n}\right)}{6} .
\end{aligned}
$$

This is the extension of Eq. (7) to the confined case. For $R \gg \lambda_{T}$, the $n=0$ term dominates the sum, yielding

$$
T>0, R \gg h, \lambda_{T}: F(R)=-\frac{3 T \alpha^{2}(0)}{\epsilon_{\mathrm{in}}^{2}(0) R^{6}} \frac{5 \gamma^{2}+\gamma^{-2}}{6},
$$

which extends Eq. (8) to the confined geometry.

Equations (10) and (12) are our central results. They account for the large-distance, retarded dispersion interaction between the confined particles at zero and finite temperature, respectively. Comparing with Eqs. (6) and (8), we see that the confinement is manifest as a factor dependent on the ratio $\gamma(0)$ between the static dielectric permittivities of the inner and outer media. This factor can be as small as 0.78 [Eq. (10)] or 0.75 [Eq. (12)], but increases indefinitely with the permittivity mismatch. The divergence of the interaction energy for $\gamma \rightarrow 0$ or $\infty$ is obviously unphysical. Although Eqs. (10) and (12) are asymptotically correct for any finite mismatch, as $\gamma$ becomes increasingly large or small one must go to ever larger interparticle distances for these asymptotes to hold. Ultimately, in the limits $\gamma \rightarrow 0, \infty$ their range of validity disappears, and the large-distance interaction obeys a different power law [16, 17]. (Detailed analysis of this behavior will be given elsewhere.) The main point, however, is that the amplification factor can be very large for reasonable values of $\gamma$. For example, for particles embedded in a polar liquid $\left[\epsilon_{\text {in }}(0)=80\right]$ which is confined by glass plates $\left[\epsilon_{\text {out }}(0)=4\right]$ at room temperature, one gets an amplification factor of about 300. If the outer medium is a gas (a free-standing film, $\epsilon_{\text {out }}=1$ ), the factor increases to about 5000 [24].

Finally, we present results from numerical integration of Eqs. (21) and (4) for two examples of practical interest. This requires expressions for $\epsilon(i \xi)$ of the various media, for which we use the Ninham-Parsegian representation [2, 25], an empirical fit based on electromagnetic absorption spectra of the materials. In the first example two polystyrene particles are confined in a slab of water between two glass plates at room temperature. The function $\epsilon_{\text {in }}(i \xi)$ for water is found in Refs. 2, 5] and that for silica glass, $\epsilon_{\text {out }}(i \xi)$, in Ref. [25]. For $\alpha(i \xi)$ we took the excess Clausius-Mossotti polarizability [4] of a polystyrene sphere, $\alpha(i \xi) / V=\left[3 \epsilon_{\text {in }}(i \xi) /(4 \pi)\right]\left[\epsilon_{\mathrm{PS}}(i \xi)-\right.$ $\left.\epsilon_{\text {in }}(i \xi)\right] /\left[\epsilon_{\mathrm{PS}}(i \xi)+2 \epsilon_{\mathrm{in}}(i \xi)\right], V$ being the particle volume and $\epsilon_{\mathrm{PS}}(i \xi)$ the permittivity of polystyrene, found in Refs. 2, 5]. The resulting potentials for two interplate separations, $h=1$ and $0.1 \mu \mathrm{m}$, are shown in Fig. 2(a) along with the unconfined potential. The interaction per volume squared has been scaled by $-T / R^{6}$. The curves, therefore, represent the effective Hamaker coefficient (divided by $\pi^{2}$, in units of $T$ ) as a function of interparticle distance. The unconfined potential clearly exhibits the crossover from the nonretarded $R^{-6}$ regime at small $R$ to the retarded $R^{-7}$ dependence at intermediate distances, and then back to the $R^{-6}$ decay due to temperature. The confined interaction deviates from the unconfined one at distances $R \gtrsim h$. The amplification factor increases moderately with distance until saturating to the asymptotic value given in Eq. (12). In the second example two polystyrene particles are confined in a hydrocarbon slab of thickness $h=3 \mathrm{~nm}$, which is embedded in water. This may mimic small hydrophobic inclusions in a biological membrane. For the oily environment we used the permittivity $\epsilon_{\text {in }}(i \xi)$ of pentane [1]. As is seen in Fig. 2(b), the amplification becomes significant only at $R \gtrsim 10 \mathrm{~h}$. This is because for smaller distances retardation has not yet set in. (Note where the unconfined potential departs from its nonretarded $R^{-6}$ behavior.)

The confinement-induced enhancement of the retarded 

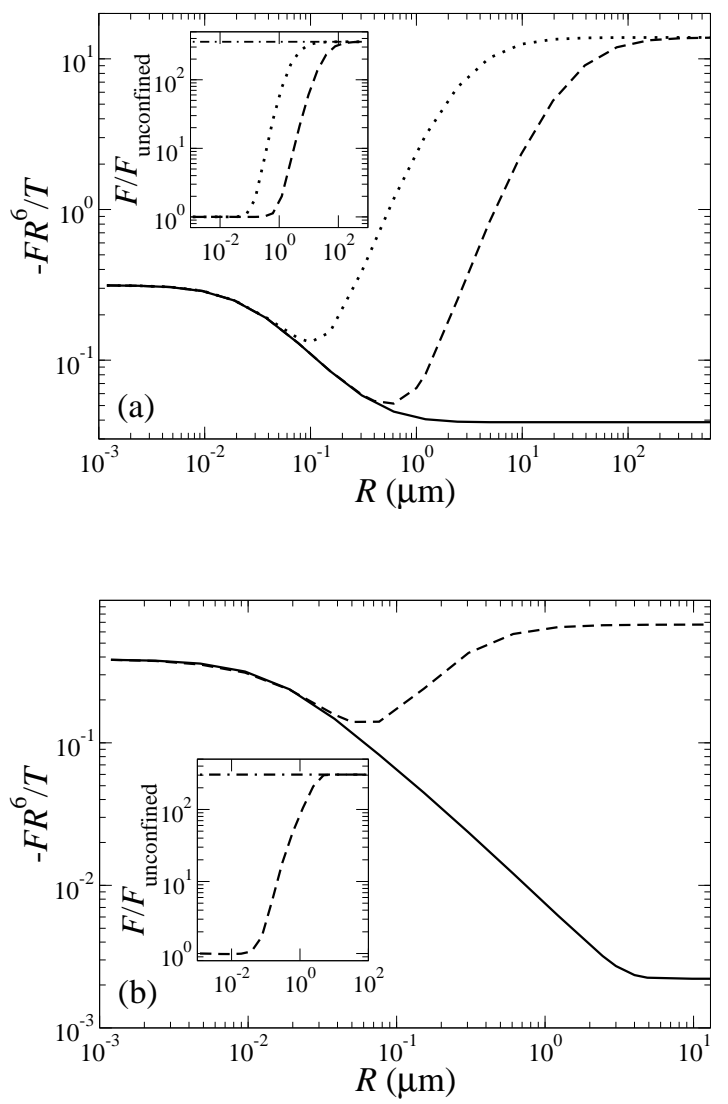

FIG. 2: Potentials of interaction as obtained from numerical integration of Eqs. (2) and (4). The interaction free energy is scaled by $-T / R^{6}$. (a) Polystyrene particles in water between two glass plates at $T=300 \mathrm{~K}$. Solid, dashed, and dotted curves correspond, respectively, to an unconfined system, $h=1 \mu \mathrm{m}$, and $h=0.1 \mu \mathrm{m}$. (b) Polystyrene particles in a hydrocarbon slab embedded in water at $T=300 \mathrm{~K}$. Solid and dashed curves correspond to an unconfined system and $h=3$ $\mathrm{nm}$, respectively. Insets in both panels present the ratio between the confined and unconfined potentials, the dash-dotted lines showing the asymptotic amplification factor of Eq. (12).

dispersion interaction can be viewed as a consequence of multiple-reflection waveguiding of the electromagnetic radiation between the boundaries. Despite the demonstrated strong effect the interaction remains weak. In the example presented in Fig. 2(a), for instance, the interaction free energy for $h=1 \mu \mathrm{m}$ and $R=10 \mu \mathrm{m}$ is about $3 \times 10^{-6} \mathrm{~T} / \mu \mathrm{m}^{6}$. Nevertheless, to achieve the same energy without confinement one would have to set the interparticle distance at about $2 \mu \mathrm{m}$. Thus, besides the fundamental significance of the strong confinement effect reported here for particle interactions in confined systems, it may become useful also in extending the range of observation of the Casimir-Polder interaction.

We benefited from discussions with D. Andelman, $\mathrm{H}$. Bary-Soroker, D. Bergman, S. Marcovitch, B. Ninham, A. Nitzan, S. Nussinov, A. Parsegian, R. Podgornik, and T. Witten. The work was supported by the Israel Sci- ence Foundation (77/03). H.D. acknowledges the Israeli Council of Higher Education (Alon Fellowship).

* Electronic address: hdiamant@tau.ac.il

[1] J. Mahanty, and B. W. Ninham, Dispersion Forces (Academic Press, London, 1976).

[2] V. A. Parsegian, Van der Waals Forces: A Handbook for Biologists, Chemists, Engineers, and Physicists (Cambridge University Press, New York, 2005).

[3] J. S. Rowlinson and B. Widom, Molecular Theory of Capillarity (Dover, Mineola, 2002).

[4] J. Israelachvili Intermolecular and Surface Forces, 2nd ed. (Academic Press, London, 1991).

[5] W. B. Russel, D. A. Saville, and W. R. Schowalter, Colloidal Dispersions (Cambridge University Press, New York, 1989).

[6] F. London, Z. Phys. 63, 245 ( 1930).

[7] H. B. G. Casimir and D. Polder, Phys. Rev. 73, 360 (1948).

[8] Note that in the retarded limit the interaction is determined by the static $(\omega \rightarrow 0)$ properties of the system.

[9] C. I. Sukenik, M. G. Boshier, D. Cho, V. Sandoghdar, and E. A. Hinds, Phys. Rev. Lett. 70, 560 (1993).

[10] S. K. Lamoreaux, Phys. Rev. Lett. 78, 5 (1997).

[11] B. W. Ninham and J. Daicic, Phys. Rev. A 57, 1870 (1998). H. Wennerström, J. Daicic, and B. W. Ninham, Phys. Rev. A 60, 2581 (1999).

[12] E. M. Lifshitz, Sov. Phys. JETP 2, 73 (1956). I. E. Dzyaloshinskii, E. M. Lifshitz, and L. P. Pitaevskii, Adv. Phys. 10, 165 (1961).

[13] L. P. Pitaevskii, Sov. Phys. JETP 10, 408 (1960).

[14] In fact, all the results depend only on the values of the dielectric functions along the imaginary axis, $\epsilon(i \xi)$ with real $\xi$ [1]. These are real, positive, monotonously decreasing functions of $\xi$ involving the imaginary part of $\epsilon(\omega)$ alone.

[15] R. Miller, T. E. Northup, K. M. Birnbaum, A. Boca, A. D. Boozer, and H. J. Kimble, J. Phys. B 38, S551 (2005).

[16] J. Mahanty and B. W. Ninham, J. Phys. A 6, 1140 (1973).

[17] M. Boström, J. J. Longdell, and B. W. Ninham, Phys. Rev. A 64, 062702 (2001).

[18] M. Cho and R. J. Silbey, J. Chem. Phys. 104, 8730 (1996).

[19] J. Mahanty and B. W. Ninham, J. Phys. A 5, 1447 (1972).

[20] C. T. Tai, Dyadic Green Functions in Electromagnetic Theory, 2nd ed. (IEEE Press, New-York, 1993).

[21] M. Avidan-Marcovitch, MSc thesis, Tel Aviv University, 2005.

[22] The limit is taken such that $R / \lambda_{T} \rightarrow 0$ but $\lambda_{0} / \lambda_{T}$ is finite. If $\lambda_{0} / \lambda_{T} \rightarrow 0$, the sum can be turned into an integral, which leads back to the zero-temperature results.

[23] A. D. McLachlan, Proc. Roy. Soc. London, Ser. A 202, 224 (1963); Mol. Phys. 6, 423 (1963).

[24] If the liquid contains mobile ions, however, the interaction will be screened beyond the wavelength corresponding to the ionic plasma frequency [5].

[25] L. Bergström, Adv. Colloid Interface Sci. 70, 125 (1997). 